\title{
Filament Tension and Phase Locking of Meandering Scroll Waves
}

\author{
Hans Dierckx, ${ }^{1}$ I. V. Biktasheva, ${ }^{2,3}$ H. Verschelde, ${ }^{1}$ A. V. Panfilov, ${ }^{1}$ and V. N. Biktashev ${ }^{3}$ \\ ${ }^{1}$ Department of Physics and Astronomy, Ghent University, 9000 Ghent, Belgium \\ ${ }^{2}$ Department of Computer Science, University of Liverpool, Liverpool L69 3BX, United Kingdom \\ ${ }^{3}$ College of Engineering, Mathematics and Physical Sciences, University of Exeter, Exeter EX4 4QF, United Kingdom \\ (Received 21 May 2017; revised manuscript received 19 September 2017; published 20 December 2017)
}

\begin{abstract}
Meandering spiral waves are often observed in excitable media such as the Belousov-Zhabotinsky reaction and cardiac tissue. We derive a theory for drift dynamics of meandering rotors in general reactiondiffusion systems and apply it to two types of external disturbances: an external field and curvature-induced drift in three dimensions. We find two distinct regimes: with small filament curvature, meandering scroll waves exhibit filament tension, whose sign determines the stability and drift direction. In the regimes of strong external fields or meandering motion close to resonance, however, phase locking of the meander pattern is predicted and observed.
\end{abstract}

DOI: 10.1103/PhysRevLett.119.258101

Introduction.-Rotating spiral waves are remarkable patterns that spontaneously occur in many spatially extended systems [1-8]. In many cases, a quasiperiodic motion of the wave pattern instead of rigid-body rotation can be recognized from the star- or flowerlike tip trajectory, shown by red traces in Fig. 1. These are called "meandering spiral waves" or "modulated rotating waves" $[9,10]$ and are observed in the Belousov-Zhabotinsky chemical reaction $[11,12]$, and in cardiac tissue experiments $[8,13]$ and numerical simulations [14-18].

The understanding of the excitation patterns exhibited by circular-core spirals in 2D, and scroll waves in 3D, has much benefited from the analysis of their motion in terms of "phase singularities," i.e., instantaneous rotation centers for the spirals, Fig. 1, and "filaments," Fig. 2, for the scrolls [19-21]. Much of the theory of meandering spiral waves has been focusing on the origin of the meander bifurcation [10,22-24], which produces epi- or hypocyclodial motion of a spiral tip, as shown in Fig. 1(a). However, meandering spirals with "linear" cores, as in Fig. 1(b), may be the building blocks of ventricular fibrillation, which motivated recent work to calculate their leading eigenmodes [25-27]. In this Letter, we derive equations of motion for biperiodic meandering 2D spirals and 3D scroll waves, without restriction to a particular shape of meander.

In 3D, it has been shown that the filament of a circularcore scroll wave is characterized by its "tension" $\gamma_{1}$, which depends on the medium parameters: $\gamma_{1}<0$ leads to evergrowing filaments $[20,28]$ if the medium is thick enough [29], resulting in a turbulent, fibrillationlike state, while

Published by the American Physical Society under the terms of the Creative Commons Attribution 4.0 International license. Further distribution of this work must maintain attribution to the author(s) and the published article's title, journal citation, and DOI. $\gamma_{1}>0$ leads to the shrinking of scroll rings, so that only filaments connecting opposite medium boundaries persist. Figure 2 shows similar behavior for meandering scroll waves. However, the applicability of the concept of filament tension to meandering scrolls has so far been a conjecture rather than fact. In this Letter, we will show when this is indeed true, and when it is not.

Methods.-We investigate spiral-shaped solutions to the reaction-diffusion system in two and three spatial dimensions under a small spatiotemporal perturbation $\mathbf{h}$ :

$$
\partial_{t} \mathbf{u}(\vec{r}, t)=\mathbf{P} \Delta \mathbf{u}(\vec{r}, t)+\mathbf{F}(\mathbf{u}(\vec{r}, t))+\mathbf{h}(\vec{r}, t),
$$

where $\mathbf{u}$ is a column matrix of state variables. Equation (1) describes both Belousov-Zhabotinsky-like chemical systems and models of cardiac tissue, depending on the choice of the diffusion constants $\mathbf{P}$ and reaction kinetics $\mathbf{F}(\mathbf{u})$. We consider two different kinetics models: the Barkley model [30], $\mathbf{u}=[u, v]^{T}, \quad \mathbf{F}=\left\{c^{-1} u(1-u)[u-(v+b / a)], u-v\right\}^{T}$, $\mathbf{P}=\operatorname{diag}(1,0), a=0.58, b=0.05, c=0.02$, Fig. 1(a),
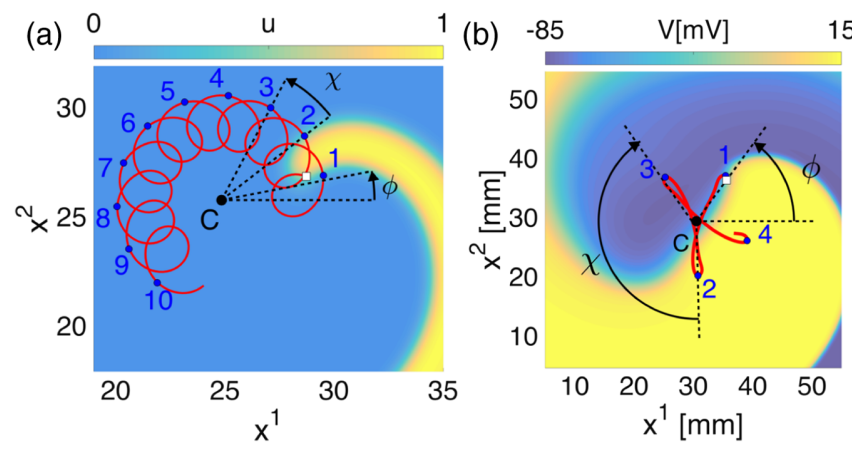

FIG. 1. Meandering spiral wave, with current tip position (white) and upcoming tip trajectory (red) for Barkley (a) and Fenton-Karma (b) kinetics. Successive "petals" (blue) are reached after time $T$ and span the angle $\chi$. 
(a)

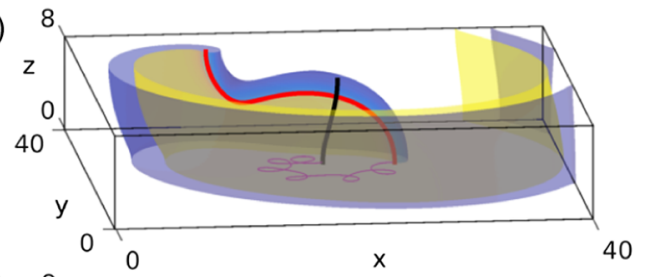

(b)

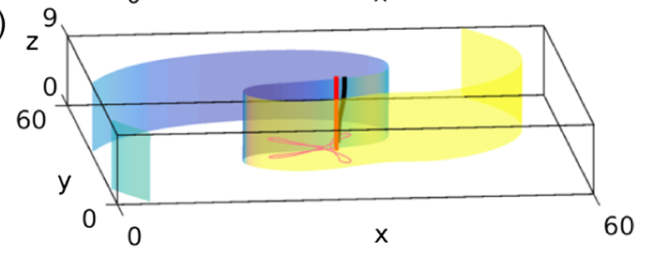

FIG. 2. Meandering 3D scroll wave evolved from a nearly straight scroll (initial filament shown in black), showing the tip trajectory on the bottom surface, current wave front (blue), wave back (yellow), and filament (red). (a) For Barkley kinetics, at $t=30$ the filament buckles and subsequently breaks up. (b) In the FK model $(t=950 \mathrm{~ms})$, the filament straightens.

and the Fenton-Karma (FK) cardiac tissue model with guinea pig parameters [17], where $\mathbf{u}=[u, v, w]^{T}, \mathbf{P}=$ $\operatorname{diag}(0.1,0,0) \mathrm{mm}^{2} / \mathrm{ms}$, Fig. 1(b).

Before presenting our formalism, we recall the classical view on meander in terms of the transition of an unperturbed $(\mathbf{h}=\mathbf{0})$ spiral from rigid to biperiodic rotation via an equivariant Hopf bifurcation [10,22-24,31]. Let $\omega_{s}$ be the angular velocity of a rigidly rotating spiral. In a frame of reference rotating with $\omega_{s}$, this spiral is a stationary solution. The corresponding linearized problem always has eigenvalues on the imaginary axis, due to the rotational $\left(\lambda_{R}=0\right)$ and translational $\left(\lambda_{T}= \pm i \omega_{s}\right)$ Euclidean symmetry of the plane. If under a parameter change another pair of eigenvalues crosses the imaginary axis at $\lambda_{H}= \pm i \Omega$, the solution becomes time periodic with period $T=2 \pi / \Omega$ in the rotating frame. The progression of the solution along this cycle can be labeled by the meander phase $\psi$; by definition, in the absence of perturbation, $\partial_{t} \psi=\Omega$.

To describe meandering spirals without relying on the proximity of the Hopf bifurcation, we note that in the lab frame, a meandering spiral is a relative periodic orbit, meaning that after the time $T$ the solution returns to the same state up to an orientation-preserving isometry $\mathcal{M}$ of the plane [10,23,32,33]. Except for the resonant case, which falls outside our present scope, $\mathcal{M}$ is a rotation by an angle $\chi$ around a point $C$. After iterating $\mathcal{M}$, the point $C$ emerges as the center of the meander pattern, see Fig. 1. By construction, $\chi$ is defined up to an integer number of full rotations. In terms of the classical approach, we can write $\chi=\omega_{s} T+2 \pi n$, for $n \in \mathbb{N}$. For our formalism, the exact choice of $\chi$ is not of principal importance. We find it convenient to demand $|\chi|<\pi$, and define $\chi$ as the (smallest) angle between consecutive petals of the tip path, see Fig. 1. Correspondingly, we consider a frame of reference rotating around $C$ with $\omega=\chi / T$, in which the solution is also $T$ periodic. As before, we define $\Omega=2 \pi / T$ and $\partial_{t} \psi=\Omega$. Note that this formalism equally holds for both cases shown in Fig. 1.

Let the angle $\phi(t)$ characterize the orientation of the steadily rotating frame (Fig. 1); by definition $\partial_{t} \phi=\omega$ for the unperturbed spiral. The transformation between the lab frame coordinates $x^{a}$ and the rotating frame coordinates $\rho^{A}$ is then $\rho^{A}=R_{a}^{A}(\phi)\left(x^{a}-X^{a}\right)$, where $R_{a}^{A}(\phi)$ is the rotation matrix over an angle $\phi$. In the rotating frame, Eq. (1) becomes

$$
\partial_{\tau} \mathbf{u}=\mathbf{P} \Delta \mathbf{u}+\omega \partial_{\theta} \mathbf{u}+\mathbf{F}(\mathbf{u}) .
$$

Here, $\tau$ is the time in that frame, $\Delta$ is the Laplacian in the $\left(\rho^{1}, \rho^{2}\right)$ plane, and $\theta$ is the polar angle in it, i.e., $\partial_{\theta}=\epsilon_{A}^{B} \rho^{A} \partial_{B}$. The unperturbed meandering spiral wave solution $\mathbf{u}_{0}\left(\rho^{1}, \rho^{2}, \psi\right)$ to Eq. (2) is $2 \pi$ periodic in $\psi$ and satisfies

$$
\mathbf{P} \Delta \mathbf{u}_{0}+\omega \partial_{\theta} \mathbf{u}_{0}-\Omega \partial_{\psi} \mathbf{u}_{0}+\mathbf{F}\left(\mathbf{u}_{0}\right)=\mathbf{0} .
$$

In what follows, we perform a standard perturbation technique used before to derive drift laws for circular-core spiral and scroll waves [19,20,34,35]. This involves linearization of Eq. (3) on $\mathbf{u}_{0}$, after which the drift caused by a perturbation $\mathbf{h}$ will be given by its projection onto the symmetry eigenmodes.

The linear operator $\hat{\mathcal{L}}$ associated with Eq. (3) is

$$
\hat{\mathcal{L}}=\hat{\mathbf{L}}-\Omega \partial_{\psi}, \quad \hat{\mathbf{L}}=\mathbf{P} \Delta+\omega \partial_{\theta}+\mathbf{F}^{\prime}\left(\mathbf{u}_{0}\right) .
$$

The operator $\hat{\mathbf{L}}$ is the same as used for the circular-core case $[19,20,35,36]$. By differentiating Eq. (3) with respect to $\rho^{1}, \rho^{2}, \theta$, and $\psi$, we find the four critical eigenmodes: $\mathbf{V}_{ \pm}=-\frac{1}{2}\left(\partial_{1} \mathbf{u}_{0} \pm i \partial_{2} \mathbf{u}_{0}\right), \mathbf{V}_{\psi}=-\partial_{\psi} \mathbf{u}_{0}, \mathbf{V}_{\phi}=-\partial_{\theta} \mathbf{u}_{0}$, where $\hat{\mathcal{L}} \mathbf{V}_{ \pm}= \pm i \omega \mathbf{V}_{ \pm}, \hat{\mathcal{L}} \mathbf{V}_{\psi}=\mathbf{0}, \hat{\mathcal{L}} \mathbf{V}_{\phi}=\mathbf{0}$. Modes $\mathbf{V}_{ \pm}$correspond to translations, $\mathbf{V}_{\phi}$ to rotations, and $\mathbf{V}_{\psi}$ to shifts in time.

To perform projections onto the symmetry modes, we use the inner products

$$
\langle\mathbf{f} \mid \mathbf{g}\rangle=\iint_{\mathbb{R}^{2}} \mathbf{f}^{H} \mathbf{g} d \rho^{1} d \rho^{2}, \quad\langle\langle\mathbf{f} \mid \mathbf{g}\rangle\rangle=\int_{0}^{2 \pi}\langle\mathbf{f} \mid \mathbf{g}\rangle \frac{d \psi}{2 \pi} .
$$

The projectors $\mathbf{W}^{M}$, also called "response functions" (RFs) in this context [37], are the critical eigenfunctions of the adjoint operator $\hat{\mathcal{L}}^{\dagger}$,

$\hat{\mathcal{L}}^{\dagger}=\hat{\mathbf{L}}^{\dagger}+\Omega \partial_{\psi}, \quad \hat{\mathbf{L}}^{\dagger}=\mathbf{P}^{H} \Delta-\omega \partial_{\theta}+\mathbf{F}^{\prime H}\left(\mathbf{u}_{0}\right)$,

such that $\hat{\mathcal{L}} \mathbf{W}^{ \pm}=\mp i \omega \mathbf{W}^{ \pm}, \hat{\mathcal{L}} \mathbf{W}^{\psi}=\mathbf{0}, \hat{\mathcal{L}} \mathbf{W}^{\phi}=\mathbf{0}$. They are $2 \pi$ periodic in $\psi$, and can be normalized as

$$
\left\langle\left\langle\mathbf{W}^{M} \mid \mathbf{V}_{N}\right\rangle\right\rangle=\delta_{N}^{M}, \quad M, N \in\{+,-, \phi, \psi\} .
$$

The biorthogonality property (7) is not practical since it involves averaging over $\psi$. However, although generally an inner product $\langle\mathbf{f} \mid \mathbf{g}\rangle$ depends on $\psi$, for products of eigenfunctions of $\hat{\mathcal{L}}$ the following Meander lemma holds (see also Refs. [26,33]):

$$
\left\langle\mathbf{W}^{M} \mid \mathbf{V}_{N}\right\rangle=\delta_{N}^{M} \quad \forall \psi, \quad M, N \in\{+,-, \phi, \psi\} .
$$


Indeed,

$$
\begin{aligned}
\Omega \partial_{\psi} & \left\langle\mathbf{W}^{M} \mid \mathbf{V}_{N}\right\rangle \\
& =\left\langle\left(\hat{\mathbf{L}}^{\dagger}-\hat{\mathcal{L}}^{\dagger}\right) \mathbf{W}^{M} \mid \mathbf{V}_{N}\right\rangle+\left\langle\mathbf{W}^{M} \mid(\hat{\mathcal{L}}-\hat{\mathbf{L}}) \mathbf{V}_{N}\right\rangle \\
& =\left(\lambda_{N}-\lambda_{M}\right)\left\langle\mathbf{W}^{M} \mid \mathbf{V}_{N}\right\rangle .
\end{aligned}
$$

If $\lambda_{M}=\lambda_{N},\left\langle\mathbf{W}^{M} \mid \mathbf{V}_{N}\right\rangle$ is constant and equal to $\left\langle\left\langle\mathbf{W}^{M} \mid \mathbf{V}_{N}\right\rangle\right\rangle=$ $\delta_{N}^{M}$. If $\lambda_{M} \neq \lambda_{N},\left\langle\mathbf{W}^{M} \mid \mathbf{V}_{N}\right\rangle(\psi)=A_{N}^{M} \exp \left[\left(\lambda_{N}-\lambda_{M}\right) \psi / \Omega\right]$. Since this is $2 \pi$ periodic, $i\left(\lambda_{N}-\lambda_{M}\right) / \Omega$ needs to be an integer. However, in the nonresonant case we have $0<|\chi|<\pi$, whence $0<|\omega / \Omega|<1 / 2$. For the critical modes, $\left(\lambda_{N}-\lambda_{M}\right) / \Omega$ thus cannot be an integer, and therefore $A_{N}^{M}=0$ and $\left\langle\mathbf{W}^{M} \mid \mathbf{V}_{N}\right\rangle=0$.

As a corollary, the instant orthogonality (8) also holds for the Cartesian basis of eigenfunctions, i.e., $M, N \in$ $\left\{\rho^{1}, \rho^{2}, \phi, \psi\right\}$ and pairs of a critical and noncritical mode.

Results.-We are now ready to calculate how a small perturbation $\mathbf{h}$, say of order $\eta$, induces spiral wave drift. Still in 2D, we decompose the exact solution to (2) as

$$
\mathbf{u}\left(\rho^{A}, \tau\right)=\mathbf{u}_{0}\left(\rho^{A}, \psi(\tau)\right)+\tilde{\mathbf{u}}\left(\rho^{A}, \tau\right),
$$

where $\tilde{\mathbf{u}}=\mathcal{O}(\eta)$ is made unique at all times by the condition $\left\langle\mathbf{W}^{M} \mid \tilde{\mathbf{u}}\right\rangle=0$ [35]. Then, we let the frame move with yet unknown perturbed velocities:

$$
\partial_{t} X^{A}=\tilde{v}^{A}, \quad \partial_{t} \psi=\Omega+\tilde{v}^{\psi}, \quad \partial_{t} \phi=\omega+\tilde{v}^{\theta},
$$

where $\tilde{v}^{M}=\mathcal{O}(\eta)$. Inserting these into Eq. (2) yields

$$
\left(\partial_{\tau}-\hat{\mathbf{L}}\right) \tilde{\mathbf{u}}-\sum_{M=\rho^{1}, \rho^{2}, \theta, \psi} \tilde{v}^{M} \partial_{M} \mathbf{u}_{0}=\mathbf{h}+\mathcal{O}\left(\eta^{2}\right) .
$$

Finally, projection onto the RFs delivers

$$
\dot{X}^{M}=v^{M}+\left\langle\mathbf{W}^{M} \mid \mathbf{h}\right\rangle+\mathcal{O}\left(\eta^{2}\right)
$$

for $M \in\{1,2, \phi, \psi\}$, where $v^{\psi}=\Omega, v^{\phi}=\omega$, and $v^{A}=0$.

The equation of motion (13) describes the spatial drift of the position $X^{1}, X^{2}$ of the center of the meander pattern, its orientation $\phi$ in the plane, and the meander phase $\psi$ of the spiral. It is a fundamental result in this Letter, as it captures the generic drift response of a meandering spiral wave to small external disturbances $\mathbf{h}(\vec{r}, t)$. Its form was stated before based on symmetry for particular cases of $\mathbf{h}$ $[24,32,38,39]$, but without the overlap integral that is necessary to quantitatively predict spiral wave drift.

In this Letter we choose to further study

$$
\mathbf{h}=\mathbf{Q} \vec{E} \cdot \nabla \mathbf{u},
$$

which has several applications. For example, in chemical systems, $\mathbf{u}$ is a vector of concentrations of reagents, and Eqs. (1) and (14) may describe the "electrophoretic" drift of spiral waves in a constant electrical field $\vec{E}$, if $\mathbf{Q}$ is the diagonal matrix of electrical mobilities of the reagents. More generically, in any reaction-diffusion system describing 3D scroll waves, one can show that the effect of diffusion in three dimensions boils down to a perturbation of the form of Eq. (14), with $\mathbf{Q}=\mathbf{P}$ and $\vec{E}=k \vec{N}$, where $k$ is the geometrical curvature of the scroll wave filament (i.e., the $3 \mathrm{D}$ extension of the rotation centers $C$ ) and $\vec{N}$ is the local normal vector to it [19].

The resulting spiral and scroll wave dynamics can for both applications mentioned above be found by substituting Eq. (14) into the general law of motion (13). Here, we will assume that the RFs $\mathbf{W}^{M}$ are essentially localized (as shown numerically in Refs. [25-27]) within an area of size $d$ and that the spatial scale over which the fields $\vec{E}$ vary is larger than $d$. In the lab frame of reference, this delivers

$$
\begin{aligned}
\partial_{t} \phi & =\omega+Q_{A}^{\phi}(\psi) R_{a}^{A}(\phi) E^{a}, \\
\partial_{t} \psi & =\Omega+Q_{A}^{\psi}(\psi) R_{a}^{A}(\phi) E^{a}, \\
\partial_{t} X^{b} & =R_{B}^{b}(\phi) Q_{A}^{B}(\psi) R_{a}^{A}(\phi) E^{a}
\end{aligned}
$$

with $Q_{A}^{M}=\left\langle\mathbf{W}^{M}|\mathbf{Q}| \partial_{A} \mathbf{u}_{0}\right\rangle$. In the case where $\vec{E}=k \vec{N}$, $\mathbf{Q}=\mathbf{P}$, system (15) describes the evolution of the scroll wave filament position $X^{b}$ in every plane locally orthogonal to the filament. We have thus generalized Keener's law of motion [19]. The main difference is that the coefficients $Q_{A}^{M}$ depend on the meander phase $\psi$.

The law of motion (15) can be simplified considerably by averaging it over several meander periods. This is most easily seen using Fourier series:

$$
\begin{aligned}
Q_{A}^{M}(\psi) & =\sum_{k \in \mathbb{Z}}{ }^{K} Q_{A}^{M} e^{i k \psi}, \\
R_{a}^{A}(\phi) & =\frac{1}{2}\left(\delta_{a}^{A}+i \epsilon_{a}^{A}\right) e^{i \phi}+\text { c.c. },
\end{aligned}
$$

where c.c. is the complex conjugate.

The dynamics of the center of the meander flower in each cross section perpendicular to the filament is then

$$
\partial_{t} X^{b}=\sum_{\ell=-2}^{2} \sum_{k \in \mathbb{Z}} F_{\ell, k}^{b} e^{i \ell \phi} e^{i k \psi}+\mathcal{O}\left(E^{2}\right) .
$$

We note that unless in resonance the set $\{|\ell \omega+k \Omega| \mid \ell \in$ $\{0, \pm 1, \pm 2\}, k \in \mathbb{Z}\}$ has a strictly positive minimal element, say $\omega_{\min }$. Then, all nonconstant terms in Eq. (17) will oscillate at a frequency of at least $\omega_{\min }$.

However, the sole constant term $F_{0,0}^{b}$ in the right-hand side of Eq. (17) will induce a constant drift velocity:

$$
\begin{aligned}
F_{0,0}^{b} & ={ }^{0} Q_{A}^{B} \frac{E^{a}}{4}\left(\delta_{B}^{b}+i \epsilon_{B}^{b}\right)\left(\delta_{a}^{A}+i \epsilon_{a}^{A}\right)+\text { c.c. } \\
& =\frac{1}{2} \overline{Q_{A}^{A}} E^{b}+\frac{1}{2} \epsilon_{B}^{A} \overline{Q_{A}^{B}} \epsilon_{a}^{b} E^{a} .
\end{aligned}
$$

In vector notation, this result can be written as

$$
\vec{V}=\Gamma_{1} \vec{E}+\Gamma_{2} \overrightarrow{\mathcal{T}} \times \vec{E},
$$


where $\vec{V}$ is the net drift motion of the filament, $\overrightarrow{\mathcal{T}}$ is the unit tangent to the filament for 3D scroll waves, and $\overrightarrow{\mathcal{T}}=\vec{e}_{z}$ for 2D spiral waves in the $X Y$ plane. From Eq. (18), the drift components parallel and perpendicular to the applied external field $\vec{E}$ are given by

$$
\begin{aligned}
& \Gamma_{1}=\overline{Q_{A}^{A}}=\frac{1}{2}\left\langle\left\langle\mathbf{W}^{A}|\mathbf{Q}| \partial_{A} \mathbf{u}_{0}\right\rangle\right\rangle, \\
& \Gamma_{2}=\frac{1}{2} \epsilon_{B}^{A} \overline{Q_{A}^{B}}=\frac{\epsilon_{B}^{A}}{2}\left\langle\left\langle\mathbf{W}^{B}|\mathbf{Q}| \partial_{A} \mathbf{u}_{0}\right\rangle\right\rangle .
\end{aligned}
$$

The time-averaged equation of motion for meandering spiral waves (19) exhibits the same dynamics as in the circular-core case. If $\mathbf{h}$ describes diffusive coupling in the third spatial dimension $(\vec{E}=k \vec{N}, \mathbf{Q}=\mathbf{P})$, Eq. (19) happens to reduce to the circular-core result from Ref. [20]:

$$
\vec{V}=\Gamma_{1} k \vec{N}+\Gamma_{2} k \vec{B},
$$

where $\vec{N}$ and $\vec{B}$ are the normal and binormal vectors to the filament. Then, we can interpret $\Gamma_{1}$ and $\Gamma_{2}$ as the scalar and pseudoscalar filament tension. Since Eqs. (19) are the laws of motion for the filament of a meandering scroll wave, it follows from Ref. [20] that the period-averaged filament length increases monotonically in time if $\Gamma_{1}<0$ and decreases if $\Gamma_{1}>0$.

To validate our results, we have determined the coefficients $P_{A}^{M}(\psi)$ for the Barkley and FK kinetics by applying $\vec{E}$ for a short time interval at different values of the meander phase $\psi$, see Sec. B of the Supplemental Material [40] for details of the numerics. Averaging $P_{A}^{B}(\psi)$ over one period delivered $\Gamma_{1}=-3.97, \Gamma_{2}=0.70$ for Barkley kinetics and $\Gamma_{1}=0.455, \Gamma_{2}=0.302$ for FK kinetics. Theoretical predictions (19) and (21) using the measured $\Gamma_{1,2}$ are in good agreement with the observed drift of spirals in a constant field $E$, and with circular scroll ring dynamics, see Fig. 3.

Since the chosen parameters in Barkley kinetics yield $\Gamma_{1}<0$, the filament will undergo Euler buckling beyond a critical thickness, as we have already seen in Fig. 2(a). The FK model has $\Gamma_{1}>0$, and Fig. 2(b) shows that a transmural filament indeed relaxes to the minimal length.

Until now, it was assumed that perturbations are small and $\omega$ is not. As noted already in Refs. [39,41], if either
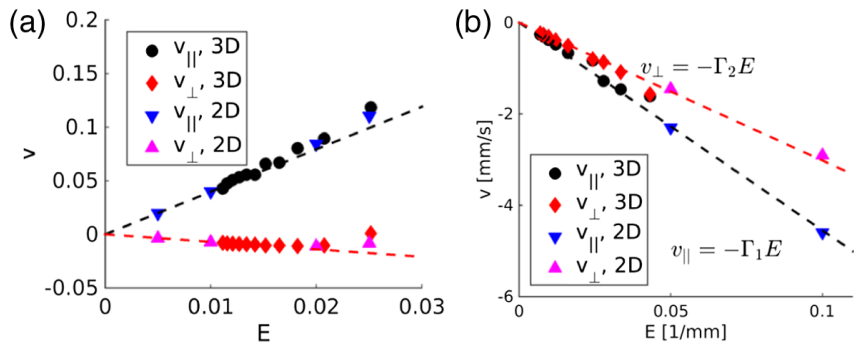

FIG. 3. Comparison of drift velocity components with theory, in the Barkley (a) and FK model (b). "3D" refers to a scroll ring simulation with $\mathbf{h}=\mathbf{P}(1 / r) \partial_{r} \mathbf{u}$. condition is broken, phase-locking between spiral rotation and its meandering may happen. We are now in a position to describe this phenomenon quantitatively. For Barkley kinetics as in Fig. 1(a) where $\omega=0.08 \ll \Omega=1.25$, one finds a qualitatively different tip trajectory when $E=$ $|\vec{E}| \geq 0.04$, see Fig. 4(a). From the first of Eqs. (15), one can show similarly to Ref. [42] that a necessary condition for locking the rotation phase is

$$
E>E_{\text {crit }}=\omega / Q, \quad Q=\sqrt{\left(\overline{Q_{1}^{\phi}}\right)^{2}+\left(\overline{Q_{2}^{\phi}}\right)^{2}} .
$$

The locked rotation angle will be $\phi_{\ell}=\arccos (-\omega / Q)-$ $\arctan \left(\overline{Q_{2}^{\phi}} / \overline{Q_{1}^{\phi}}\right)$. Given the computed $Q_{A}^{\phi}$, expression (22) predicts $E_{\text {crit }}=0.041$, closely matching the value of 0.04 found in Fig. 4(a). Figure 4(b) shows a comparison for different values of the parameter $a$; it can be seen that the Arnold tongue for phase-locking is well described by Eqs. (22).

In the $(a, b)$ parameter space of Barkley's model, phaselocking is found near the line of resonant meander. Already for a field strength of $E=0.03$, Fig. 4(c) shows phaselocking in a significant portion of the meander region, where it leads to relatively large drift velocities [see Fig. 4(d)]. In qualitative terms, Fig. 4(a) shows that the meander flower opens up during phase-locking, and the resulting drift speed is therefore close to the mean "orbital velocity" $\omega R$ of the tip along the meander flower, where $R$ is the time-averaged radius of the meander flower. This result does not contradict Eq. (15) since when $\omega \rightarrow 0$, the center of the rotating frame is far away. One can instead use a different rotating frame, with the origin shifted to the average tip position. This gives, in leading order,
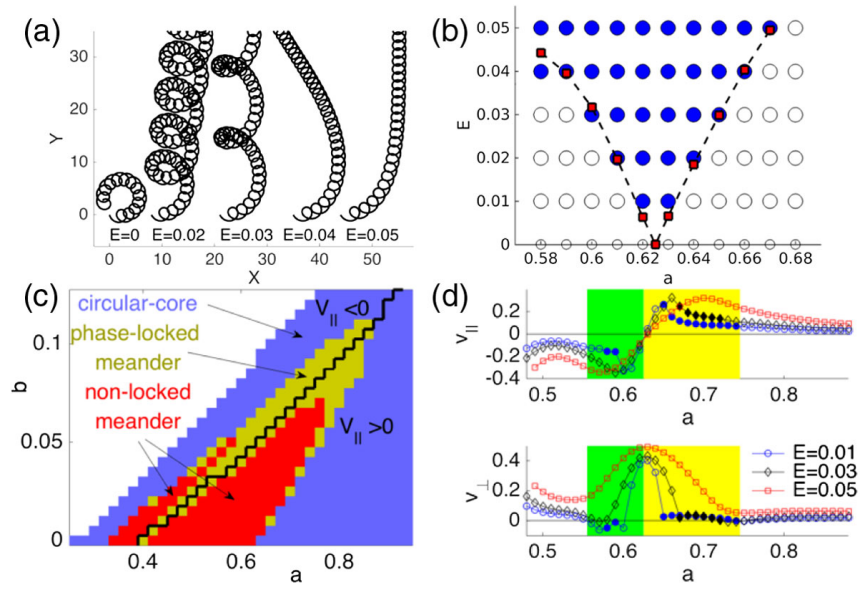

FIG. 4. Phase-locking in Barkley's model. (a) Drift trajectories with $\vec{E}=E \vec{e}_{x}$ for parameters as in Fig. 1(a), showing phaselocking when $E>0.04$. (b) Arnold tongue confirming the theoretical prediction in Eq. (22). (c) Occurrence of phaselocking for $E=0.03$ in (a),(b) parameter space with $c=0.02$. (d) Drift components parallel and perpendicular to $E$, for $b=0.05$. The colored background indicates meander. 
$V_{\|}(E)=\omega R \cos \left[\phi_{\ell}(E)\right], \quad V_{\perp}(E)=\omega R \sin \left[\phi_{\ell}(E)\right]$.

We noted that the curve $V_{\|}=0$ closely matches the locus of resonant meander. We have however not found analytical proof of this property, and a counterexample in the LuoRudy-I cardiac tissue model is known [43].

Discussion.-One motivation for this work was to see how the concept of filament tension generalizes to meandering scroll waves. Only when averaged over many meander periods does the dynamics reduce to the circular-core case. The tension concept has already been used for meander in cardiological literature [13] and modeling studies [43]. Here, we have shown that the emerging property of filament tension does indeed explain the (in)stability of scroll waves in simple cases, such as those in Fig. 2. Real heart tissue is more complicated in many respects. For instance, a significant phenomenon is pinning to heterogeneities. Some pinning effects have been described before using perturbation methods [44-46]. Thin domains of irregular thickness $L(x, y)$ (e.g., the cardiac wall) can also be captured by Eq. (15), with $\mathbf{h}=\mathbf{P} \nabla \ln L$ [46].

On short time scales, the dynamics is much more complex and the concept of filament tension cannot be applied. The orientation of the meander pattern may phaselock to external fields, and thickness or parameter gradients.

In general, the theory that was presented here opens the pathway to analyzing and predicting the trajectory and stability of meandering spiral and scroll waves in reactiondiffusion media of diverse nature.

H. D. was funded by FWO-Flanders during part of this work. The computational resources (Stevin Supercomputer Infrastructure) and services used in this work were provided by the VSC (Flemish Supercomputer Center), funded by Ghent University, FWO and the Flemish Governmentdepartment EWI. I. V. B. and V. N. B. gratefully acknowledge EPSRC (UK) support via Grant No. EP/D074789/1. I. V. B. acknowledges EPSRC (UK) support via Grant No. EP/P008690/1. V. N. B. acknowledges EPSRC (UK) current support via Grant No. EP/N014391/1 (UK).

[1] W. Jahnke, Ch. Henze, and A. T. Winfree, Nature (London) 336, 662 (1988).

[2] F. Siegert and C. J. Weijer, Proc. Natl. Acad. Sci. U.S.A. 89, 6433 (1992).

[3] J. Lechleiter, S. Girard, E. Peraltal, and D. Clapham, Science 252, 123 (1991).

[4] N. A. Gorelova and J. J. Bures, J. Neurobiol. 14, 353 (1983).

[5] M. A. Allessie, F. I. M. Bonke, and F. J. G. Schopman, Circ. Res. 33, 54 (1973).

[6] R. A. Gray, A. M. Pertsov, and J. Jalife, Nature (London) 392, 75 (1998).
[7] F. X. Witkowsky, L. J. Leon, P. A. Penkoske, W. R. Giles, M. L. Spano, W. L. Ditto, and A. T. Winfree, Nature (London) 392, 78 (1998).

[8] M. Haissaguerre et al., Circulation 130, 530 (2014).

[9] A. T. Winfree, Chaos 1, 303 (1990).

[10] D. Barkley, Phys. Rev. Lett. 72, 164 (1994).

[11] A. T. Winfree, Science 181, 937 (1973).

[12] O. Steinbock, V.S. Zykov, and S.C. Müller, Nature (London) 366, 322 (1993).

[13] M. Yamazaki, S. Mironov, C. Taravant, J. Brec, L. M. Vaquero, K. Bandaru, U. M. R. Avula, H. Honjo, I. Kodama, O. Berenfeld, and J. Kalifa, Cardiovasc. Res. 94, 48 (2012).

[14] I. R. Efimov, V. I. Krinsky, and J. Jalife, Chaos, Solitons Fractals 5, 513 (1995).

[15] M. Courtemanche, R. J. Ramirez, and S. Nattel, Am. J. Physiol. 275, H301 (1998).

[16] V. N. Biktashev and A. V Holden, Proc. R. Soc. B 263, 1373 (1996).

[17] F. H. Fenton and A. Karma, Chaos 8, 20 (1998).

[18] A. Bueno-Orovio, E. M. Cherry, and F. H. Fenton, J. Theor. Biol. 253, 544 (2008).

[19] J. P. Keener, Physica (Amsterdam) 31D, 269 (1988).

[20] V. N. Biktashev, A. V. Holden, and H. Zhang, Phil. Trans. R. Soc. A 347, 611 (1994).

[21] R. H. Clayton, E. A. Zhuchkova, and A. V. Panfilov, Prog. Biophys. Molec. Biol. 90, 378 (2006).

[22] D. Barkley, Phys. Rev. Lett. 68, 2090 (1992).

[23] V. N. Biktashev, A. V. Holden, and E. V. Nikolaev, Int. J. Bifurcation Chaos Appl. Sci. Eng. 06, 2433 (1996).

[24] M. Golubitsky, V. G. LeBlanc, and I. J. Melbourne, J. Nonlinear Sci. 7, 557 (1997).

[25] C. D. Marcotte and R. O. Grigoriev, Chaos 25, 063116 (2015).

[26] C. D. Marcotte and R. O. Grigoriev, Chaos 26, 093107 (2016).

[27] H. Dierckx, H. Verschelde, and A. V. Panfilov, Chaos 27, 093912 (2017).

[28] A. V. Panfilov and A. N. Rudenko, Physica (Amsterdam) 28D, 215 (1987).

[29] H. Dierckx, H. Verschelde, O. Selsil, and V. N. Biktashev, Phys. Rev. Lett. 109, 174102 (2012).

[30] D. Barkley, Physica (Amsterdam) 49D, 61 (1991).

[31] E. V. Nikolaev, V. N. Biktashev, and A. V. Holden, Int. J. Bifurcation Chaos Appl. Sci. Eng. 09, 1501 (1999).

[32] V. G. LeBlanc and C. Wulff, J. Nonlinear Sci. 10, 569 (2000).

[33] A. J. Foulkes and V. N. Biktashev, Phys. Rev. E 81, 046702 (2010).

[34] V. N. Biktashev and A. V. Holden. Chaos, Solitons Fractals 5, 575 (1995).

[35] H. Verschelde, H. Dierckx, and O. Bernus, Phys. Rev. Lett. 99, 168104 (2007).

[36] I. V. Biktasheva, D. Barkley, V. N. Biktashev, G. V. Bordyugov, and A. J. Foulkes, Phys. Rev. E 79, 056702 (2009).

[37] I. V. Biktasheva and V. N. Biktashev, Phys. Rev. E 67, 026221 (2003).

[38] C. Wulff, Ph. D. thesis, Freie Universität Berlin, 1996.

[39] V. G. LeBlanc, Nonlinearity 15, 1179 (2002). 
[40] See Supplemental Material at http://link.aps.org/ supplemental/10.1103/PhysRevLett.119.258101 for details.

[41] S. Grill, V. S. Zykov, and S. C. Müller, J. Phys. Chem. 100, 19082 (1996).

[42] B.-W. Li, M.-C. Cai, H. Zhang, A. V. Panfilov, and H. Dierckx, J. Chem. Phys. 140, 184901 (2014).

[43] S. Alonso and A. V. Panfilov, Chaos 17, 015102 (2007).
[44] V. N. Biktashev, D. Barkley, and I. V. Biktasheva, Phys. Rev. Lett. 104, 058302 (2010).

[45] D. Pazo, L. Kramer, A. Pumir, S. Kanani, I. Efimov, and V. Krinsky, Phys. Rev. Lett. 93, 168303 (2004).

[46] I. V Biktasheva, H. Dierckx, and V. N. Biktashev, Phys. Rev. Lett. 114, 068302 (2015). 